\title{
Variable renewal rate and growth properties of cell populations in colon crypts
}

\author{
A. Di Garbo, ${ }^{1}$ M. D. Johnston, ${ }^{2}$ S. J. Chapman, ${ }^{3}$ and P. K. Maini ${ }^{2,4}$ \\ ${ }^{1}$ Istituto di Biofisica CNR, Via G. Moruzzi 1, 56124 Pisa, Italy \\ ${ }^{2}$ Centre for Mathematical Biology, Mathematical Institute, University of Oxford, Oxford, United Kingdom \\ ${ }^{3}$ Oxford Centre for Industrial and Applied Mathematics, Mathematical Institute, University of Oxford, Oxford, United Kingdom \\ ${ }^{4}$ Oxford Centre for Integrative System Biology, Department of Biochemistry, University of Oxford, Oxford, United Kingdom
}

(Received 27 May 2009; revised manuscript received 5 March 2010; published 4 June 2010)

\begin{abstract}
A nonlinear mathematical model is used to investigate the time evolution of the cell populations in colon crypts (stem, semidifferentiated and fully differentiated cells). To mimic pathological alteration of the biochemical pathways leading to abnormal proliferative activity of the population of semidifferentiated cells their renewal rate is assumed to be dependent on the population size. Then, the effects of such perturbation on the population dynamics are investigated theoretically. Using both theoretical methods and numerical simulations it is shown that the increase in the renewal rate of semidifferentiated cells strongly impacts the dynamical behavior of the cell populations.
\end{abstract}

DOI: 10.1103/PhysRevE.81.061909

PACS number(s): 87.18.Fx, 87.15.A-, 87.10.-e, 87.17.Aa

\section{INTRODUCTION}

The gut is anatomically divided into two compartments: the small intestine and the colon, both of which are lined with a single layer of epithelial cells. This epithelium is the fastest self-renewing tissue of adult mammals: on average it is replaced every 5-6 days. The epithelium of both compartments is organized into invaginations called crypts. In addition the small intestine contains fingerlike protrusions called villi that are absent in the colon. The fast renewal rate of gut epithelium is sustained by stem cells residing in the bottom of the crypts. Stem cells at the base of the crypt generate the semidifferentiated (or transit amplifying) cells that fill the lower part of the crypt and divide several times before giving rise to the fully differentiated cells. The fully differentiated cells migrate upwards and fill the upper part of the crypt before being shed into the gut lumen $[1,2]$. There is experimental evidence suggesting that this process is a combination of population pressure (due to proliferation) and active cell migration $[3,4]$. A schematic representation of the cell compartments of the colonic crypt is shown in Fig. 1.

The primary driving force underlying the proliferation of epithelial cells is the Wnt signaling pathway, which is highly conserved in the animal kingdom $[1,2,5]$. In the absence of the Wnt ligand the concentration of the $\beta$-catenin protein is maintained at physiological levels due to the activity of a degradation complex. This complex consists of adenomatous polyposis coli (APC), the glycogen synthase kinase $3 \beta$, the casein kinase I and regulates the $\beta$-catenin phosphorylation status $[1,2,5]$. When the Wnt ligand is present the destruction complex is inactivated and $\beta$-catenin is not phosphorylated and accumulates in the cell $[1,2,5]$. In such conditions $\beta$-catenin is able to reach the cell nucleus and interact with the T-cell factor/lymphoid enhancer factor (TCF/LEF) family of transcription factors to promote gene expression. The activation of TCF/LEF is an important factor underlying the proliferative activity of healthy gut epithelium as well as the pathological tissue activity occurring in colorectal cancer $[1,2,5]$.

To investigate stem cell dynamics and cancer several models have been introduced [6-19] and an overview and classification of them was presented in [13]. One of the earliest and most influential models of colorectal cancer is that of Tomlinson and Bodmer [6] consisting of three compartments: stem cells, semidifferentiated cells, and fully differentiated cells. This model is discrete and stem and differentiated cells are assumed either to die or to differentiate or self-renew at the end of their cell cycle. It was shown that the alteration of the rates of programmed cell death or cell differentiation can explain several aspects of tumor behavior [6]; this study was extended and generalized in $[17,18]$ to account for feedback and to remove the restrictions of synchrony and common cell cycle time in all compartments implicitly assumed in the original model. In Boman et al. [7] a compartmental model was used to understand the experimental data on patients with familial adenomatous polyposis (FAP). In [11] a three compartment model of colorectal cancer was investigated: the first compartment describes stem cells, the second contains the semidifferentiated cells and the last one the fully differentiated cells. It was found that mutations in the compartment of semidifferentiating cells are crucial for colon cancer initiation. Similarly, by using a linear model of colon cancer, it was shown that chromosomal instabilities are a key factor for tumorigenesis [10]. By using a similar approach these results were extended to leukemia stem cells [16]. With the aid of a continuous mathematical model describing stem, early and late progenitor cells in neural cancer it was found that oncogenic events and sudden cell population depletion strongly impact the proliferative activity of the cells [12]. The effects of genetic mutation on a population of stem cells were also studied in [14]. As discussed the Wnt signaling pathway is an important factor in the development of a cancerous tissue and the biochemical processes driving this signaling cascade were studied theoretically in [15]. By using a mathematical model describing the stem, transit amplifying and fully differentiated cells of the olfactory epithelium it was found that the self-regulation of the proliferative rates of these cells can enhance local stability [19]. The understanding of the basic cellular mechanisms underlying cancer generation is a very important scientific challenge and, at present, is still an open problem for most cancers [20]. Complexity in carcinogenesis occurs at 


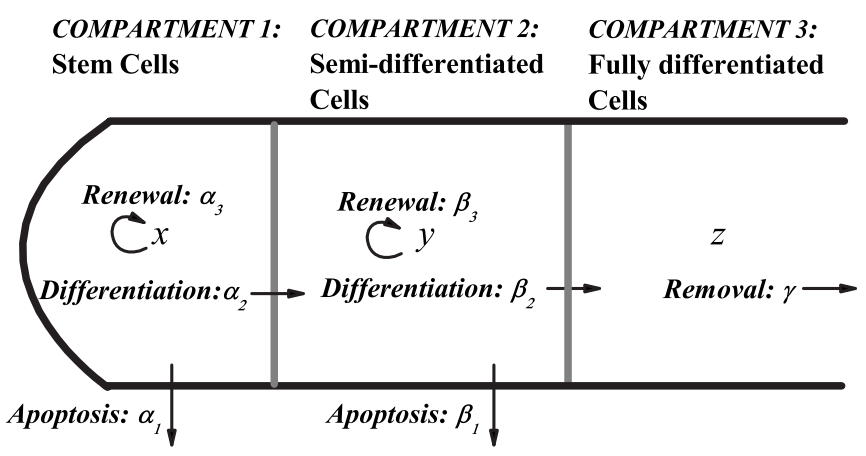

FIG. 1. Schematic representation of the colonic crypt structure adopted in the model. There are three compartments and the arrows indicate the processes driving the cell behavior. See text for further details.

many hierarchical levels involving DNA, RNA, proteins, intracellular and extracellular biochemical pathways, tissues, and organs. Therefore it is important to stress that the model that will be studied in the present paper neglects the internal structure of the cells but aims to understand how properties at the cell level affect tissue level behavior.

In our paper a mathematical model describing the cell populations in colorectal crypts is introduced and studied. In particular the conditions leading to unlimited growth of the cell populations are investigated. The aim is to understand how self-renewal, differentiation, and apoptosis affect the size of the involved cellular species. For simplicity, the model consists of three compartments in which the spatial location of the cells is not considered so the model is not suited to investigate the mechanisms controlling cell migration or other connected phenomena. The study of the more complex cell population dynamics, arising from the inclusion of spatial cell location and migration phenomena, will be pursued in future work. However, as pointed out in [13], compartmental models are very useful for obtaining insight on the processes driving the population dynamics of the crypt, in spite of the above approximation. An additional comment on our model is that it neglects the heterogeneous expression of cells during tumor progression: i.e., the nonuniform distribution of the microscopic state of the cells $[21,22]$. Most tumors become more aggressive and malignant in their characteristics over time although this time course may be quite variable; this phenomenon is termed tumor progression [21]. As pointed out by Nowell [21] it is common, particularly with rapidly growing tumors, to find heterogeneity so that some subpopulations of the neoplastic cells may appear much further advanced than others in the degree of tumor progression. As predicted by Nowell [23] this phenomenon is a consequence of the clonal evolution process. Here we use a macroscopic model, in terms of ordinary differential equations, in which the above mechanisms driving tumor evolution are not described. However it is possible to introduce models at a lower scale in which cellular heterogeneity, arising from tumor progression, can be taken into account [22]. Such approaches are described in a general setting in [22].

The starting point of the present work is the model of the colorectal crypt recently proposed by some of us [17], de- scribing three cellular species: the stem cells, the semidifferentiated cells and the fully differentiated cells. The goal of the present paper is to generalize that work, and to this aim we will consider a model in which the self-renewal rate of semidifferentiated cells is an increasing function of the population size. The stability properties of the corresponding stationary states will be studied to identify those dynamical regimes which can lead to unlimited growth. In the first part of the paper the study will be carried out theoretically, while in the final part the results of model simulations will be discussed. Moreover, the effect of noise on the dynamical behavior of the cell populations will be investigated.

\section{MODEL DESCRIPTION}

Let $x$ denote the population of stem cells, $y$ denote that of the semidifferentiated (or transit-amplifying) cells, and $z$ denote that of the fully differentiated cells in the colonic crypt. Let us consider the continuum model describing these populations adopted in Johnston et al. [17]. In this model the population of stem cells is described by the equation $\dot{x}$ $=\alpha_{3} x-\alpha_{2} x-\alpha_{1} x$, where the parameter $\alpha_{3}$ is the renewal rate of these cells, $\alpha_{2}$ is the transition rate from the stem cell compartment to the semidifferentiated cell compartment, and $\alpha_{1}$ represents the rate of stem cell apoptosis (see Fig. 1). Similarly, the population of semidifferentiated cells is described by the equation $\dot{y}=\beta_{3} y-\beta_{2} y-\beta_{1} y+\alpha_{2} x$, where $\beta_{3}$ is the renewal rate of these cells and $\beta_{1}$ the corresponding apoptotic rate, while $\beta_{2}$ is the transition rate from the semidifferentiated cell compartment to that of the fully differentiated cells (see Fig. 1). The time evolution of the population of the fully differentiated cells is described by the equation $\dot{z}=-\gamma z+\beta_{2} y$, where $\gamma$ is the removal rate of these cells (see Fig. 1). To summarize, the full model is

$$
\begin{gathered}
\frac{d x}{d t}=\left(\bar{\alpha}-\alpha_{2}\right) x, \\
\frac{d y}{d t}=\left(\bar{\beta}-\beta_{2}\right) y+\alpha_{2} x, \\
\frac{d z}{d t}=-\gamma z+\beta_{2} y .
\end{gathered}
$$

where $\bar{\alpha}=\alpha_{3}-\alpha_{1}$ and $\bar{\beta}=\beta_{3}-\beta_{1}$. It was shown that nontrivial positive steady state solutions of Eqs. (1a)-(1c) can exist only in the cases $\bar{\alpha}-\alpha_{2}<0, \bar{\beta}-\beta_{2}=0$ and $\bar{\alpha}-\alpha_{2}=0, \bar{\beta}-\beta_{2}$ $<0$ [17]. This system is, of course, structurally unstable. To obtain a more realistic description of the dynamics of the stem cells the previous model is modified by introducing the term $-k x^{2}(k>0)$ on the right-hand side of Eq. (1a). Thus, the time evolution of the stem cell population is described by logistic dynamics [24]. To justify this modification some general considerations are necessary. Both the normal and cancer tissue contain the same population of cells: the stem cells, the semidifferentiated cells, and the fully differentiated cells [2]. Normal tissue renewal and growth of cancer are both due to semidifferentiated cells. Usually, the stem cells 
of both normal and cancer tissues are relatively few in number (compared to the semidifferentiated cells and fully differentiated cells) and are proliferating [2]. Experimental results obtained in Hydra stem cells clearly show that the population size is controlled by negative feedbacks from the neighboring cells: i.e., the increase in the population size determines a decrease in the self-renewal rate of the stem cells [25]. The dependence of the renewal rate on the stem cell density was modeled by a logistic dynamics by Hardy and Stark [8] and by a sigmoid profile by Zhdanov [26]. More recently the problem of cell population growth was addressed in a general framework by Kilian et al. [27]. Based on general biological principles these authors formulated general equations describing the growth curves of all cell types by showing that these processes are driven by logistic dynamics [27]. Moreover, by using experimental data on the in vitro proliferation of mesenchymal stem cells, it was found that the time evolution of the population size is described by a logistic equation [28]. Moreover, a logistic kinetics to describe the growth of a population of stem cells was also adopted by other authors $[14,29,30]$.

Normal tissue renewal and growth of cancer both occur by division of the transit amplifying (or semidifferentiated) cells [2]. Moreover, experimental data from a variety of animal and tissue culture studies suggest that tumor growth decelerates with increasing cell population size and sigmoidal (or S-shaped) curves are used to fit the data [31-34]. Thus, the second modification takes into account that the rate of renewal of the population of the semidifferentiated cells depends on the population size. Such an assumption is also in keeping with the recent work of d'Onofrio and Tomlinson [18] and can be ascribed, for example, to the alteration of the mechanisms regulating $\beta$-catenin concentration [2]. The latter can arise from transcription errors or through a loss of the function of the APC degradation factor (see Sec. I) $[35,36]$. Thus, on the basis of the above discussion, it is assumed that the rate of self-renewal of the semidifferentiated cells is a sigmoidal function of the population size, in particular a Hill-like growth rate is adopted:

$$
\beta_{3}(y)=\beta_{3}+\frac{p y^{n}}{q+y^{n}}, \quad p>0, \quad q>0, \quad n \in \aleph .
$$

Inserting the above change into Eq. (1b) leads to the following system of equations to describe the time evolution of the cell populations in the colon crypts:

$$
\begin{gathered}
\frac{d x}{d t}=\left(\bar{\alpha}-\alpha_{2}\right) x-k x^{2}, \\
\frac{d y}{d t}=\left(\bar{\beta}-\beta_{2}\right) y+\frac{p y^{n+1}}{\left(q+y^{n}\right)}+\alpha_{2} x, \\
\frac{d z}{d t}=-\gamma z+\beta_{2} y .
\end{gathered}
$$

Because the population size of the stem cells follows a logistic growth toward a stationary value [2,27,28,31-34], a positive value of the parameter $\bar{\alpha}-\alpha_{2}$ is adopted in this paper. From Eq. (3b) it follows that unlimited growth occurs if $\bar{\beta}-\beta_{2}=\beta_{3}-\beta_{2}-\beta_{1}>0$. On the other hand the experimental data on hematopoietic cells of cat and mouse discussed in [37] indicate that the value of $\bar{\beta}-\beta_{2}$ can be positive or negative and we allow for this in the model. A similar assumption was also adopted in other studies of cell population dynamics in the colon crypt $[17,38]$. For instance, changes in $\beta$ could arise because of cellular damage, the mutation of genes controlling the differentiation process, alteration of the Wnt signaling pathways, a change in the apoptosis rate, etc. [2].

\section{STATIONARY STATES}

To simplify the notation let us define $\alpha=\bar{\alpha}-\alpha_{2}$ and $\beta$ $=\bar{\beta}-\beta_{2}$ in Eqs. (3a) and (3b). Let us remark that Eq. (3a), describing the stem cell population, is not coupled to the remaining two and is completely solvable. If at time $t=0$ the size of the population of stem cells is $x_{0}>0$, then its solution is given by $x(t)=\left(\alpha x_{0} k^{-1}\right) /\left[x_{0}+\left(\alpha / k-x_{0}\right) e^{-\alpha t}\right]$. Because $\alpha$ $>0$ it follows that the nontrivial stationary state $\left(x_{e q}=\alpha / k\right)$ of Eq. (3a) is stable. Before proceeding to analyze the full system [Eqs. (3a)-(3c)] let us show that solutions exist and are positive. The right-hand sides of Eqs. (3a)-(3c) are continuous with continuous partial derivatives and this implies the existence and uniqueness of the solution of the initial value problem. As shown before $x(t)=\left(\alpha x_{0} k^{-1}\right) /\left[x_{0}+(\alpha / k\right.$ $\left.\left.-x_{0}\right) e^{-\alpha t}\right]>0$, thus it is sufficient to show that $y(t)>0$ and $z(t)>0$. To this aim it is sufficient to show that by choosing arbitrary initial conditions $x(0)=x_{0}>0, y(0)=y_{0}>0$, and $z(0)=z_{0}>0$, the solutions to Eqs. (3b) and (3c) are such that $y(t)>0$ and $z(t)>0$. Consider first the equations $\dot{\bar{y}}=\beta \bar{y}$ and $\dot{\bar{z}}=-\gamma \bar{z}$ satisfying the initial conditions $\bar{y}(0)=y_{0}>0$ and $\bar{z}(0)=z_{0}>0$. The solutions to these equations are obviously positive. Since $y_{0}>0$ and $z_{0}>0$ it is possible to choose a three-dimensional ball, $B_{0}$, centered at $\left(x_{0}, y_{0}, z_{0}\right)$ entirely lying in the region $x>0, y>0$ and $z>0$. Then there exists a time $T_{1}>0$ such that for $t<T_{1}$ the solution of Eqs. (3a)-(3c) with initial condition $\left(x_{0}, y_{0}, z_{0}\right)$ is entirely contained in $B_{0}$. From this it follows that for $t<T_{1}, p y^{n+1} /\left(q+y^{n}\right)+\alpha_{2} x>0$ and $\beta_{2} y>0$ and since $\dot{y}(t) \geq \dot{\bar{y}}$ and $\dot{z}(t) \geq \dot{\bar{z}}$, this implies that $y(t) \geq \bar{y}(t)>0$ and $z(t) \geq \bar{z}(t)>0$ for $t<T_{1}$. Moreover, since $\dot{y}(t)>0$ and $\dot{z}(t)>0$ it follows that $y\left(T_{1}\right)>y_{0}$ and $z\left(T_{1}\right)>z_{0}$. Now, let us set $x_{1}=x\left(T_{1}\right)>0, y_{1}=y\left(T_{1}\right)>0$, and $z_{1}=z\left(T_{1}\right)$ $>0$ as the new initial conditions and let us consider the ball $B_{1}$ centered at the point $\left(x_{1}, y_{1}, z_{1}\right)$. Then, the above protocol can be repeated with the new initial conditions to find the new initial conditions $x_{2}=x\left(T_{2}\right)>0, y_{2}=y\left(T_{2}\right)>0$, and $z_{2}$ $=z\left(T_{2}\right)>0$. This operation can be performed an arbitrary number of times and this implies the positivity of the solutions of Eqs. (3a)-(3c).

The stationary states of Eqs. (3a)-(3c) are determined by finding the solutions of the following set of equations:

$$
\begin{gathered}
\alpha x-k x^{2}=0, \\
\beta y+\frac{p y^{n+1}}{\left(q+y^{n}\right)}+\alpha_{2} x=0,
\end{gathered}
$$




$$
\gamma z-\beta_{2} y=0 .
$$

From Eq. (4a) it follows that the equilibrium values of the population of stem cells are $x_{e q}=0$ or $x_{e q}=\alpha / k$. Let $x_{e q}=0$, then from Eqs. (4b) and (4c) it follows that $y_{e q}=z_{e q}=0$ is one possible solution. As $\alpha>0$ it follows that the stationary state $S=\left(x_{e q}, y_{e q}, z_{e q}\right)=(0,0,0)$ is unstable. Let us now search for stationary states such that $x_{e q}=0$ and $y_{e q} \neq 0$. The equilibrium values of the population of semidifferentiated cells $(y)$ are the roots of the equation $\beta+p y^{n} /(q$ $\left.+y^{n}\right)=0$. Let $\beta \geq 0$, then in this case there are no roots in the interval $] 0,+\infty[$, as $p, q>0$. Biologically realistic solutions exist only when $-p<\beta<0$ : in this case the equilibrium value of the population of semidifferentiated cells is $y_{e q}=[-\beta q /(\beta+p)]^{1 / n}$. As before, the corresponding stationary state $S_{2}=\left\{0,[-\beta q /(\beta+p)]^{1 / n}, \beta_{2} y_{e q} / \gamma\right\}$ is unstable.

Let us now assume that $x_{e q}=\alpha / k$. Substituting in Eq. (4b) we obtain the following equation:

$$
P(y)=y^{n+1}(\beta+p)+R y^{n}+\beta q y+R q=0,
$$

where $R=\alpha \alpha_{2} / k>0$. For each positive root $\left(y^{*}\right)$ of this polynomial the corresponding stationary state of Eqs. (3a)-(3c) is $S=\left(x_{e q}, y_{e q}, z_{e q}\right)=\left[\alpha / k, y^{*},\left(\beta_{2} y^{*}\right) / \gamma\right]$. To discuss the existence of the positive roots of $P(y)$ Descartes' rule of signs is used.

$$
\text { (A) Let }(\beta+p)>0 \text {. }
$$

The signs of the coefficients are

$$
\begin{array}{ccccc}
y^{n+1} & y^{n} & y & R q & \beta \text {-values } \\
+ & + & + & + & \beta>0 \\
+ & + & - & + & -p<\beta<0 .
\end{array}
$$

It follows that in the case $-p<\beta<0$ there are 2 or 0 positive roots, while no positive roots exist for $\beta>0$. To investigate the local stability properties of the corresponding stationary states the sign of the time derivative of the population of semidifferentiated cells will be studied in a neighborhood of the equilibrium value $\left(y^{*}\right)$. Obviously, if $y^{*}$ is an unstable fixed point then the corresponding stationary state of Eqs. (3a)-(3c) will also be unstable. Moreover it can be proved that all stationary states for which $\left.\frac{d P(y)}{d y}\right|_{y=y^{*}} \neq 0$ are hyperbolic. Then the Hartman-Grobman theorem [39] implies that the linearly stable (or unstable) states are also locally nonlinearly stable (or unstable).

Let us list the properties of the polynomial $P(y)$ defined in Eq. (5): $P(0)=R q>0$ and $\lim _{y \rightarrow-\infty} P(y)=+\infty$ for odd $n$ values, while $\lim _{y \rightarrow-\infty} P(y)=-\infty$ for even $n$ values. Moreover $\lim _{y \rightarrow+\infty} P(y)=+\infty$ for any $n$. From the above properties it follows that for odd $n$ values and when two positive roots exist, the corresponding graph of $P(y)$ is like that depicted in panel a of Fig. 2. Then, from the plot of Fig. 2(a), it follows that the stationary state corresponding to $S_{1}$ is stable while that corresponding to $S_{2}$ is unstable (see the arrows). This case is interesting because an unlimited growth of the population of semidifferentiated cells can occur. This can happen by choosing initial conditions within the basin of attraction of $S_{2}$ or in the presence of a suitable perturbation. For instance, let us assume that the populations are in a dynamical a)
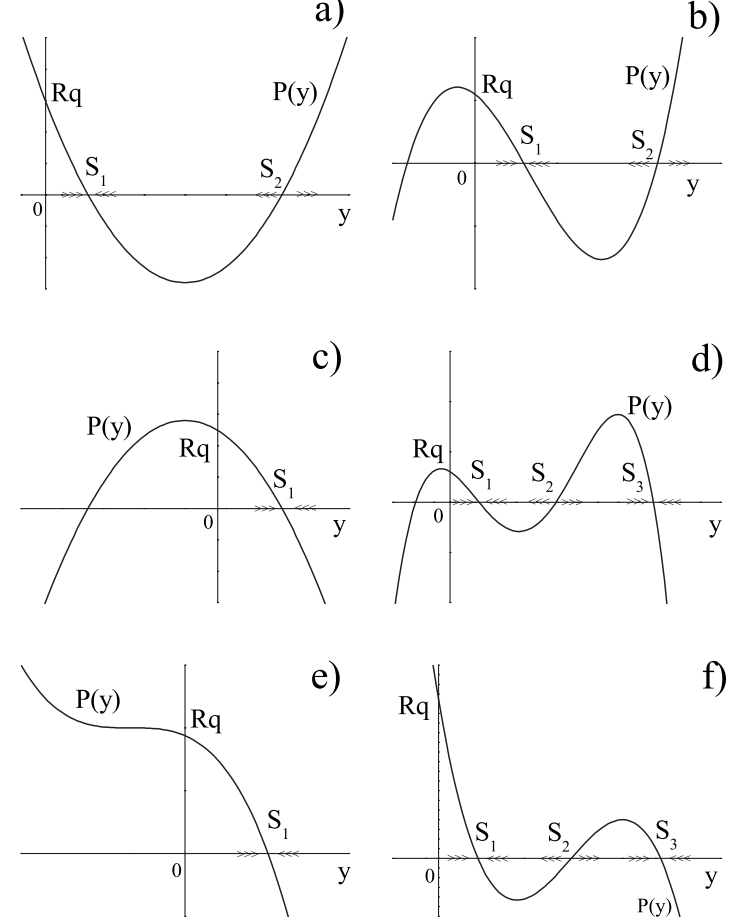

FIG. 2. Possible plots of polynomial $P(y)$ [Eq. (5)] for different parameter values. Panel (a): odd $n$ values and $-p<\beta<0$; panel (b): even $n$ values and $-p<\beta<0$. Panel (c): odd $n$ values, $\beta<-p$ with a single positive root; panel (d): odd $n$ values, $\beta<-p$ with three distinct roots. Panel (e): even $n$ values, $\beta<-p$ with a single root; panel (f): even $n$ values, $\beta<-p$ with three distinct roots.

regime close to the stationary state $S_{1}$. Then, the presence of a suitable perturbation can bring the system into the basin of attraction of the unstable state $S_{2}$ and unlimited growth can occur. Such behavior will be illustrated in more detail later by using specific numerical simulations. Similarly, for even $n$ values the generic graph of $P(y)$, when two positive roots exist, is like that shown in panel b of Fig. 2. The root $S_{1}$ corresponds to a stable stationary state and $S_{2}$ to an unstable one. Also in this case we can have unlimited growth. The case $\beta+p=0$ is equivalent to the previous one: only 2 or 0 roots can exist and the stability properties of the corresponding stationary states are the same as above.

Before continuing the study of the stationary states, it is necessary to make some remarks concerning the role of multistability in a biological context. There is experimental evidence that bistability plays an important role in the determination of the cell fate options that include self-renewal, differentiation, apoptosis, and lineage specification. The experimental data suggest that during development or differentiation cells make very precise transitions between stable network states (or gene expression patterns) [40]. For instance, these transitions can occur when the level of a single transcription factor within a genetic network is subject to fluctuations [40-46]. Such changes can be driven by the internal noise that is inherent to the biochemical reactions or to external noise originating from random variations of the external signaling conditions $[40,41,45]$. For instance Wang et al. [47] studied how human mesenchymal progenitor cells (transit-amplifying cells or semidifferentiated cells) under- 
went differentiation: it was found that this process exhibits nonlinear features similar to those characterizing the functioning of a bistable switch model [47]. Thus the authors suggest that the differentiation process is not a linear process and can be described by a nonlinear bistable switch. Moreover to model the experimental data, a S-shaped function was used to describe the nonlinear positive feedback loop characterizing the differentiation process. In another recent paper the contribution of gene expression noise to the phenotypic cell-to-cell variability within clonal populations was studied experimentally [48], suggesting that, although the mechanisms underlying cell-to-cell variability are not well known, this phenomenon exhibits multistability and noisedriven transitions between stable states is one source of cellto-cell clonal population heterogeneity [48]. How the noise impacts the behavior of our model will be investigated numerically in the final section of this paper. Coming back to the analysis of the stationary states of Eqs. (3a)-(3c) let us consider now the case $\beta+p<0$. It will be shown, as the experimental data suggest, that our model predicts the existence of bistable regimes when such condition is fulfilled.

$$
\text { (B) Let }(\beta+p)<0 \text {. }
$$

In this case the signs of the coefficients of the polynomial $P(y)$ are

$$
\begin{array}{ccccc}
y^{n+1} & y^{n} & y & R q & \beta-\text { values } \\
- & + & - & + & \beta<-p .
\end{array}
$$

There are three variations and the number of positive roots of $P(y)$ can be 3,1 , or 0 . Moreover, $\lim _{y \rightarrow-\infty} P(y)=-\infty$ for odd $n$ values, while $\lim _{y \rightarrow-\infty} P(y)=+\infty$ for even $n$ values. Lastly, $\lim _{y \rightarrow+\infty} P(y)=-\infty$ for any $n$ value. For odd $n$ values the graph of the polynomial $P(y)$, when a single positive root exists, is like that shown in Fig. 2(c). In this case, from $d y / d t=\beta y+\frac{p y^{n+1}}{q+y^{n}}+R=P(y) /\left(q+y^{n}\right)$, it follows that the stationary state of Eqs. (3a)-(3c) corresponding to $S_{1}$ is globally stable for biologically realistic initial conditions. When $P(y)$ possesses three positive roots its graph looks like that shown in Fig. 2(d) and the stationary states corresponding to $S_{1}$ and $S_{3}$ are stable, while that corresponding to $S_{2}$ is unstable. Also in this case unlimited growth cannot occur. The same conclusions also hold for even $n$ values (panels e and $\mathrm{f}$ of Fig. 2).

Overall, the above findings imply that unlimited growth can occur when the parameter $\beta$ satisfies the following inequality: $-p<\beta<0$. A biological interpretation of the previous condition is the following. As $\beta=\beta_{3}-\beta_{2}-\beta_{1}$ the condition $\beta>-p$ is equivalent to $\beta_{3}+p>\beta_{2}+\beta_{1}$, and the last inequality states that unlimited growth can occur because the maximum value of the renewal rate of the population of semidifferentiated cells is larger than the sum of the differentiation and apoptotic rates. The above condition implies that there exists a threshold value $y_{t h}$ separating two different dynamical regimes. For $0<y(t)<y_{t h}$ the population of semidifferentiated cells is bounded. When $y(t)>y_{t h}$ unlimited growth occurs. This conclusion can be proved as follows. As $\beta=\beta_{3}-\beta_{2}-\beta_{1}<0$, then from Eq. (2) it follows that the inequality $\beta_{3}(y)>\beta_{2}+\beta_{1}$ will be satisfied if $y>y_{t h}=\left[\frac{-q \beta}{p+\beta}\right]^{1 / n}$.
Thus, if the size of the population of the semidifferentiated cells overcomes the threshold then unlimited growth occurs. An important point that deserves a mention is the relevance of the condition $p>\beta+p>0$ within the biological context. There is clear experimental evidence that the self-renewal rate of stem cells and semidifferentiated cells is variable $[1,5,49]$. The causes of this variation can be intrinsic (levels of transcription factors, signaling pathways, etc.) or extrinsic (host factors, microenvironment, immune response, etc.) [5]. For instance mutation of the p53 gene that is involved in apoptosis may be an important factor in causing changes in the net growth rate of cells $[6,35]$. In our model the selfrenewal rate of the semidifferentiated cells is assumed to be variable as the experimental data suggest. The condition $p$ $>\beta+p>0$ is biologically realistic and can be justified, for instance, by alteration of the Wnt signaling pathways (or the p53 signaling pathways) both controlling the growth property of the intestinal epithelium [5]. For instance a decrease in the apoptosis rate $\beta_{1}$ (or the differentiation rate $\beta_{2}$ ) of the semidifferentiated cells determines an increase in the value of $\beta+p=\beta_{3}-\beta_{2}-\beta_{1}+p$. On the other hand when $\beta+p<0$ the rate of apoptosis and differentiation overcomes the rate of the positive feedback driving self-renewal and abnormal tissue growth cannot occur.

Let us investigate the boundedness properties of the solutions of Eqs. (3a)-(3c) when the stem cell population is assumed to be constant: $x=x_{e q}=\alpha / k$. The following proposition provides the conditions for bounded solutions.

Proposition I. Let $x=x_{e q}=\alpha / k$ then the solutions of Eqs. (3a) $-(3 \mathrm{c})$ are bounded if (a) $\beta=-p, n=1, R<p q$ or (b) $\beta$ $+p<0$.

Proof. These results can be proved by studying the signs of $d y / d t$ and $d z / d t$.

The biological meaning of the above proposition is as follows: when $\beta+p<0$ the rate of apoptosis and differentiation overcomes the rate of the positive feedback driving selfrenewal and abnormal tissue growth cannot occur. When $\beta$ $+p=0$ it follow that $\dot{y}=-p q y /\left(q+y^{n}\right)+R$. If $n>1$ then $\dot{y}$ $>0$ for $y>[q /(n-1)]^{1 / n}$ and the solution starting at $y(0)$ $=y_{0}>[q /(n-1)]^{1 / n}$ cannot be bounded. Instead, if $n=1$ and $R<p q$ then the solution of the above equation is bounded and an acceptable stationary value of the population of semidifferentiated cells will exist $\left(y_{e q}>0\right)$.

\section{MODEL GENERALIZATION}

Let us now consider a more general form of the model described by Eqs. (3a)-(3c) by adopting, for the rate of renewal of semidifferentiated cells, the following form:

$$
\beta_{3}(y)=\beta_{3}+F(y) .
$$

We consider which properties the function $F$ should have to be biologically realistic. As discussed in the previous sections the growth rate of solid tumors decelerates with increasing tumor size [25,34]. Therefore, the dependence of the net growth rate of the cells on the population size can be described by a sigmoidal function $[34,49]$. Whence it is assumed that the function $F(y)$ possesses the following properties: 
(i) Differentiable in $[0,+\infty[$.

(ii) $F(0)=0$.

(iii) $F^{\prime}(y)>0$.

(iv) $\lim _{y \rightarrow+\infty} F(y)=L<+\infty$.

The model now reads as

$$
\begin{gathered}
\frac{d x}{d t}=\alpha x-k x^{2}, \\
\frac{d y}{d t}=\beta y+F(y) y+\alpha_{2} x, \\
\frac{d z}{d t}=-\gamma z+\beta_{2} y .
\end{gathered}
$$

As in Sec. II, existence and positivity of solutions for positive initial conditions can easily be shown. Moreover, the justification for using logistic dynamics to describe the stem cell compartment is as that in Sec. II. Let us now search for the stationary states of this dynamical system. From the first equation it follows that $x_{e q}=0$ or $x_{e q}=\alpha / k$;

$$
\text { (A) } x_{e q}=0 \text {. }
$$

It can be proved that in this case biologically realistic stable stationary solutions of Eqs. (9a)-(9c) do not exist;

$$
\text { (B) } x_{e q}=\frac{\alpha}{k} \text {. }
$$

By substituting $x=x_{e q}=\frac{\alpha}{k}$ we obtain the following equation:

$$
\beta y+F(y) y+R=0,
$$

where $R=\left(\alpha_{2} \alpha\right) / k>0$. This equation is not satisfied by $y$ $=0$, thus we search for roots within the interval $] 0,+\infty[$. Equation (10) can be rearranged to yield the equivalent equation:

$$
F(y)=-\left(\frac{R}{y}+\beta\right) .
$$

As $F(y)>0$ for $y \in] 0,+\infty[$ it follows that positive roots of Eq. (11) can exist only if $\beta<0$. Let us now prove the following proposition.

Proposition II. Let $F(y)$ be a differentiable function on $[0,+\infty[$ satisfying the following properties:

(i) $F(0)=0$.

(ii) $F^{\prime}(y)>0$.

(iii) $\lim _{y \rightarrow+\infty} F(y)=L<+\infty$.

Let be $R>0$ and $\beta<0$ and $L<-\beta$, then the function $F(y)+\left(\frac{R}{y}+\beta\right)$ has at least one positive root.

The proof of this proposition is given in Appendix A. We remark that the requirement $L<-\beta$ is a necessary condition for the proof of the above proposition. In fact there are cases (with $L>-\beta$ ) where, although the function $F(y)$ satisfies all the hypotheses of the proposition, a positive root can or cannot exist. For instance let us consider the functions $F(y)$ $=\left(0.6 y^{2}\right) /\left(y^{2}+160\right)$ and $-(\beta+R / y)=-(3 / y-0.59)$, for which the inequality $L=0.6>-\beta=0.59$ holds. In this case the equation $F(y)=-(\beta+R / y)$ possesses two positive roots. However, if the choice $F(y)=\left(0.6 y^{2}\right) /\left(y^{2}+20\right)$ is adopted, then the pre-

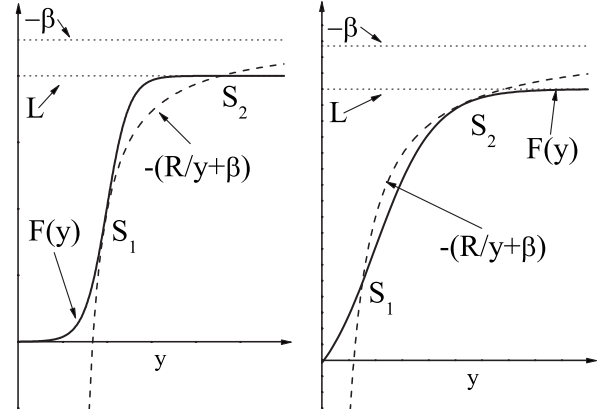

FIG. 3. Plots of the function $F(y)$ (thick black line) and $-(R / y+\beta)$ (dash black line) when the total number of roots is $N$ $=2$ and $L<-\beta$. See text for details.

vious equation does not possess any positive root.

Let us now assume that the function $F(y)+\left(\frac{R}{y}+\beta\right)$ possesses only a single root $\left(y^{*}\right)$. Then the following proposition characterizes the stability property of the stationary state of Eqs. (9a)-(9c) corresponding to $y^{*}$.

Proposition III. Let $y^{*}>0$ be the only positive root of the function $F(y)+(R / y+\beta)$, with $F(y)$ satisfying the properties of proposition II and $R>0, \beta<0, L<-\beta$. Then the stationary state $S_{0}=\left(x_{e q}, y_{e q}, z_{e q}\right)=\left(\frac{\alpha}{k}, y^{*}, \frac{\beta_{2} y^{*}}{\gamma}\right)$ is stable.

The proof of this proposition is given in Appendix B.

We now investigate the stability properties of the corresponding stationary states when Eq. (12) possesses $N>1$ distinct positive roots $y_{j}^{*}\left(j=1,2, \ldots, N, y_{j}^{*}<y_{j+1}^{*}\right)$. For $N=2$, $\beta<0, L+\beta<0$ the possible graphical configurations of the functions $F(y)$ and $-(R / y+\beta)$ are those shown in Fig. 3.

For the case depicted in the left panel the stationary state corresponding to $S_{2}$ is stable. For the stationary state corresponding to $S_{1}$ one of the eigenvalues of the Jacobian matrix of Eqs. (9a) $-(9 \mathrm{c})$ is zero: $\lambda_{2}=\beta+\frac{d}{d y}[y F(y)]_{y=y^{*}}=0$. In this case the stability of this stationary state can be investigated by using the local properties of the graphs of $F(y)$ and $-(R / y+\beta)$. As the sign of $\dot{y}$ is positive both to the left and to the right of $S_{1}$ it follows that this stationary state is not stable. By using the same approach it can be shown that for the configuration reported on the right panel of Fig. 3 unlimited growth cannot occur. This happens because $S_{1}$ is stable and $\dot{y}<0$ both to the left and right of $S_{2}$.

The existence of more than two distinct zeros can also occur, depending on the choice of $F(y)$. Let $y_{j}^{*}$ $\left(j=1,2, \ldots, N, y_{j}^{*}<y_{j+1}^{*}\right)$ be the $N>2$ distinct roots, then the following proposition holds.

Proposition $I V$. If unlimited growth of the population of semidifferentiated cells occurs then the stationary state corresponding to $y_{N}^{*}$ is unstable.

The proof of this proposition is given in Appendix C. The boundedness properties of the solutions of these equations are characterized by the following proposition.

Proposition $V$. Let $F(y)$ satisfy all properties specified in proposition II and $x=x_{e q}=\alpha / k$. If $\beta+L<0$ then all solutions of Eqs. (9b) and (9c) are bounded.

The proof of this proposition is given in Appendix D. We remark that in the case $\beta+L=0$ and $x=x_{e q}=\alpha / k$ the boundedness properties of the solutions of Eqs. (9b) and (9c) are dependent on the choice of $F(y)$. 
To take into account the possible biological implications of the above findings it is important to make some appropriate comments. An important biological concept characterizing cell biology is that of cell fate options. It is thought that cells initiate differentiation, apoptosis, or self-renewal depending on the level of activation of specific biochemical signaling pathways. The molecular processes underlying the switch from one state to another determines (through the activation of suitable genes) the cell fate decision [40]. The relevance of switchlike decisions in biological processes has been revealed in a wide range of systems [40,50]. For instance in [51] it was shown that the activation of many molecular processes can be described by sigmoidal functions which allow the biological system the possibility of adopting alternative stable steady states. Moreover, positive feedback loops—-such as the one used in our model $[\beta y+F(y) y]$-are at the basis of the complex biochemical pathways controlling cell fate decisions [51]. The multistable nature of biological switches is very important for the determination of cell fate in unicellular and multicellular organisms [52-55]. Thus, the change in the levels of gene expression can switch a cell from one stable state to another. Moreover there are clear experimental and theoretical data indicating that multistability arises in biological systems controlled by positive feedback loops $[40,44,56]$. In the case of colorectal tissue such stable stationary states could be associated with the presence of adenomas. Our model suggests the existence of multistability in the colorectal epithelium and these states can be associated with the presence of different levels of adenomatous tissue in line with the above discussion.

Another question to be asked is the following: which biological conditions are required in order that Eqs. (9a)-(9c) possess a single stable stationary state? It can be proven that if $0<L<-\beta$ and $d F / d y<R / y^{2}$ then only one steady state exists. From a biological point of view this, together with proposition $\mathrm{V}$, indicates that unlimited growth of the colorectal epithelium tissue cannot occur when $0<L<-\beta$. The biological meaning of the above condition can be inferred from the definition of $\beta=\beta_{3}-\beta_{2}-\beta_{1}$ : in fact $0<L<-\beta$ implies $L+\beta_{3}-\beta_{2}-\beta_{1}<0$. The last condition has a very simple biological interpretation: when the maximum value of the rate of renewal $\left(L+\beta_{3}\right)$ of the semidifferentiated cells is smaller than the sum of the apoptosis and differentiation rate then unlimited growth cannot occur. Moreover, let us show that in this case the model prediction can be interpreted as the appearance of a benign colon polyposis. In fact, when $F(y)$ $=0$ and $\beta<0$ the stable stationary state of Eqs. (9a)-(9c) is $S=\left(\frac{\alpha}{k},-\frac{R}{\beta},-\frac{\beta_{2} R}{\gamma \beta}\right)$. Instead, when $F(y) \neq 0$ and $0<L<-\beta$, the new stable equilibrium solution of Eqs. (9a)-(9c) is $S$ $=\left(\frac{\alpha}{k}, y^{*},-\frac{\beta_{2}}{\gamma} y^{*}\right)$, with $y^{*}>-(R / \beta)$. This means that in the new stable stationary state the population size of the semidifferentiated and fully differentiated cells is bigger than $\left(-\frac{R}{\beta}-\frac{\beta_{2} R}{\gamma \beta}\right)$.

We now investigate the biological insights gained from the above mathematical study. When considering the colonic crypts we first must establish whether a stationary state (i.e., homeostasis) can be achieved and if this state is stable. In the model, when $F(y)=0$, this condition is represented by the stable steady state $S=\left(\frac{\alpha}{k},-\frac{R}{\beta},-\frac{\beta_{2} R}{\gamma \beta}\right)$. The development of col- orectal cancer is widely believed to occur through the adenoma to carcinoma transformation $[5,36]$. The loss of Wnt signaling is the early event in the formation of the adenoma. The complete inactivation of the APC complex causes the formation of multiple bowel adenomas leading to a strong increase in the probability of developing colorectal cancer $[5,36]$. In our model the alteration of tissue growth arising from the above conditions can be achieved with the introduction of an additional contribution to the renewal rate of the semidifferentiated cells represented by $F(y) \neq 0$. Whence, if propositions II and III hold simultaneously, a stable stationary state can exist only if the net proliferation rate $(L+\beta)$ of semidifferentiated cells satisfies $\beta+L<0$. In this case the corresponding biological interpretation of the theoretical results is that there will be the formation of an adenoma without an aberrant and unlimited tissue growth. When there are more stationary states (that, from a biological point of view, can be interpreted as the formation of multiple bowel adenomas $[5,36])$ the situation becomes more complex and the condition to avoid unlimited growth is given in proposition $\mathrm{V}$. Therefore, if $\beta+L<0$ our results suggest that in the presence of multiple bowel adenomas the transition to carcinoma cannot occur. This conclusion is biologically realistic because the net renewal rate of the cells $\left(L+\beta_{3}\right)$ is smaller than the sum of the apoptosis and differentiation rate.

The results of propositions II and III can be generalized and this is achieved by requiring weaker constraints on the properties of the function $F(y)$.

Proposition VI. Let $F(y)$ be a differentiable function defined in $\mathfrak{R}$ and $|F(y)|<M<+\infty$. If $R>0$ and $\beta<-M$, then the function $F(y)+(R / y+\beta)$ has at least one positive root.

Proof. The proof follows the same approach as that to prove proposition II. Moreover it can be shown that, when the function $F(y)+(R / y+\beta)$ has a single positive root, the corresponding stationary state of Eqs. (9a)-(9c) is stable.

The boundedness properties of the corresponding solutions are defined by the following proposition.

Proposition VII. Let $F(y)$ be a differentiable function defined in $\mathfrak{R}$ and $|F(y)|<M<+\infty$. If $\beta+M<0$ and $x=x_{e q}$ $=\alpha / k$ then the solutions of Eqs. (9b) and (9c) are bounded.

Proof. The proof is similar to that of proposition V.

The previous results were obtained by assuming a population dependence of the renewal rate of the semidifferentiated cells. We now consider the case when the other rates also exhibit a population dependence. Let us assume that

$$
\begin{gathered}
\beta_{3}(y)=\beta_{3}+F(y), \\
\beta_{2}(y)=\beta_{2}-H(y)>0, \\
\beta_{1}(y)=\beta_{1}-K(y)>0,
\end{gathered}
$$

where the function $\phi(y)[\phi(y) \equiv F(y), H(y), K(y)]$ satisfies the properties described in proposition II or VI.

By substituting Eqs. (12a)-(12c) into Eq. (9b) it follows: 


$$
\frac{d y}{d t}=\beta y+Z(y) y+\alpha_{2} x, \quad[Z(y)=F(y)+H(y)+K(y)] .
$$

From the properties of the functions defining $Z(y)$, it follows that Eq. (13) is equivalent to Eq. (9b). This implies that the properties of the corresponding dynamical system are equivalent to those already discussed.

\section{EFFECTS OF NOISE ON THE DYNAMICS OF CELL POPULATIONS}

In this section the effects of noise on the dynamical behavior of the cell populations will be investigated. In particular, Gaussian white noise is added to the right-hand side of Eq. (3b) describing the time evolution of the population of semidifferentiated cells, which now reads $\dot{y}=\beta y+\frac{p y^{n+1}}{q+y^{n}}+\alpha_{2} x$ $+\sigma \xi(t)$, where $\sigma$ is the noise amplitude and $\xi(t)$ is a Gaussian stochastic process of zero mean and unit standard deviation. This noisy perturbation is introduced in the system to mimic random fluctuations arising from internal and external noise. We motivate the introduction of the noise by noting that the control of the cellular processes is regulated by the levels of gene transcription and the corresponding fluctuations are the main source of the internal noise of the cell $[41,48,57,58]$. The external noise arises from fluctuations of specific factors such as cell-cell interactions, levels of signaling molecules, rate constants of particular biochemical reactions, etc. $[41,59]$. Our aim is to understand how these random fluctuations impact the dynamics of the cell populations. In the simulations the parameter values used are within the range of values adopted by other authors $[6,18,38]$. Moreover the parameter values were selected in order to represent the main dynamical regimes described in the theoretical analysis carried out in Sec. III.

Let us consider the case corresponding to the plot of the polynomial $P(y)=y^{n+1}(\beta+p)+R y^{n}+\beta q y+R q, \quad\left(R=\alpha \alpha_{2} / k\right)$ reported in panel a of Fig. 2. For the adopted parameter values $\alpha=0.5, \quad \alpha_{2}=0.1, k=0.1, \quad \beta=-0.3, p=0.35, q=50$, $n=3$ the two roots are $y_{1}^{*} \cong 1.97, y_{2}^{*} \cong 3.37$. The stationary state $S_{1}=\left(\alpha / k, y_{1}^{*}, \beta_{2} y_{1}^{*} / \gamma\right)$ is linearly stable, while $S_{2}$ $=\left(\alpha / k, y_{2}^{*}, \beta_{2} y_{2}^{*} / \gamma\right)$ is unstable. The top panel of Fig. 4 shows plots describing the time evolution of the population of semidifferentiated cells with different initial conditions in absence of noise $(\sigma=0)$. When the initial conditions are close to the basin of attraction of the stationary state $S_{1}$ the time evolution of the population of semidifferentiated cells converges to $y_{1}^{*}$. When the initial conditions are chosen to fall within the basin of attraction of $S_{2}$, then unlimited growth will occur. The inset shows the plot of the corresponding polynomial $P(y)$. The estimation of the tumor doubling time from these simulations gives a value of about 80 weeks. This value is within the range of values found for colorectal cancer in human (from 72 to 232 weeks [60]).

The case described above has implications from the physiological point of view. In fact the stationary state $S_{1}$ can be interpreted as the normal homeostatic state in which all cell populations are in equilibrium. Let us assume that a
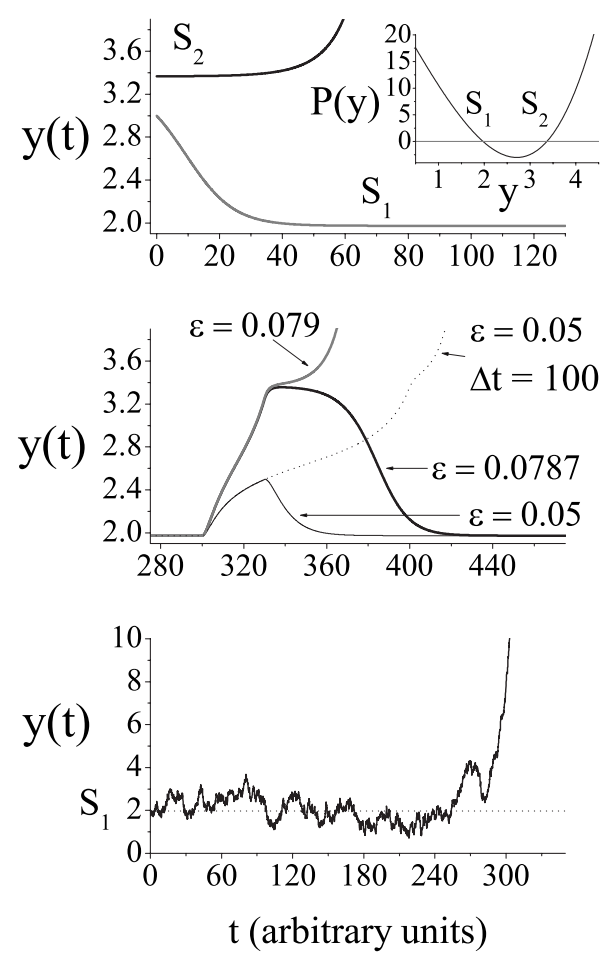

FIG. 4. Top panel: time evolution of the population of semidifferentiated cells starting with different initial conditions. In the inset is reported the plot of $P(y)$ corresponding to the following parameter values: $\alpha=0.5, \alpha_{2}=0.1, k=0.1, \beta=-0.3, p=0.35, q=50, n=3$. Middle panel: time evolution of $y(t)$ in the presence of a perturbation of amplitude $\epsilon$ and duration $\Delta t$; for the thin, thick black lines and for the gray line it is $\Delta t=30$. Bottom panel: time evolution of the population of semidifferentiated cells in the presence of a noise amplitude $\sigma=0.25$. The dotted line represents the population size at equilibrium $\left(y_{e q}=1.97\right)$. For all panels $-p<\beta<0$.

perturbation of finite duration increases the proliferative rate of the population of semidifferentiated cells. Within the physiological analogy, the perturbation acting on the dynamical system can be interpreted as arising from the alteration of some biochemical processes controlling cell proliferation. In fact, the experimental results on the study of colorectal cancers show that about $90 \%$ of them occur through an alteration of the function of the APC complex $[1,5,13,35,36,61,62]$. As discussed the APC complex plays an important role in the control of cell proliferation by regulating the concentration level of $\beta$-catenin [13]. When the APC complex does not function correctly the $\beta$-catenin accumulates and an alteration of the cell proliferation rate occurs. In particular conditions this situation can degenerate leading to the formation of cancer $[1,5,10,12,13,35,36]$. Thus, the presence of the perturbation will determine the transition $S_{1} \rightarrow S_{1}^{*}(t)$. If the nonstationary state $S_{1}^{*}(t)$ remains within the basin of attraction of $S_{1}$ then, a suitable time after the perturbation is turned off, $S_{1}^{*}(t) \rightarrow S_{1}$. Thus it is expected that if the amplitude and duration of the perturbation are below threshold, then the cells will be able to recover (due to self-regulatory mechanisms) their original equilibrium. If the threshold is exceeded the initial equilibrium cannot be restored and unlimited growth occurs. In the middle panel of 

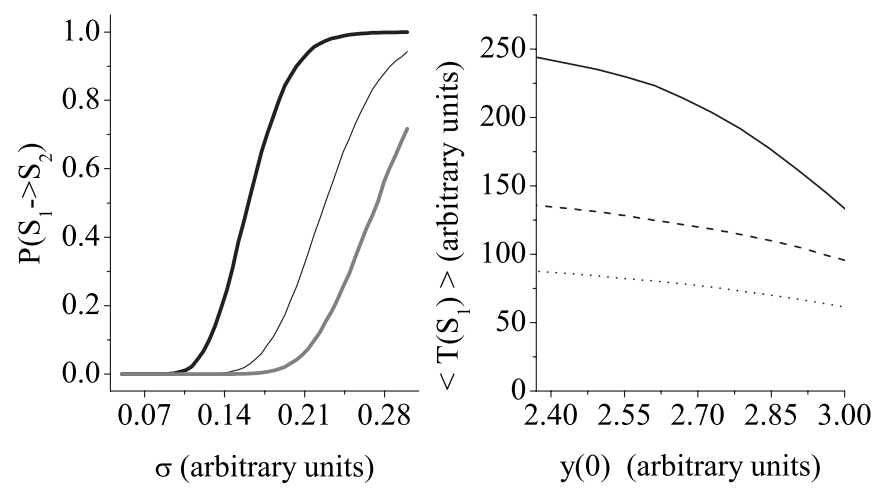

FIG. 5. The left panel shows the transition probability from $S_{1}$ to $S_{2}$ plotted against the noise amplitude $(\sigma)$ for several parameter values: $\alpha=0.5, p=0.35$ (thick black line); $\alpha=0.5, p=0.32$ (thin black line); $\alpha=0.45, p=0.35$ (gray line). The right panel shows the values of the mean exit time plotted against the initial value $[y(0)]$ of the population size of the semidifferentiated cells for different noise intensity: $\alpha=0.5, p=0.35, \sigma=0.15$ (black line); $\alpha=0.5, p$ $=0.35, \sigma=0.25$ (dashed line); $\alpha=0.5, p=0.35, \sigma=0.3$ (dotted line). For all panels $(\beta+p)<0, \beta=-0.3$, and $n=3$.

Fig. 4 are reported the results of the numerical simulations (in absence of noise) of Eqs. (3a)-(3c) showing this. In the simulation it was assumed that the perturbation affects the self-renewal rate of the semidifferentiated cells: i.e., $\beta_{3}(y)$ $=\beta_{3}+\frac{p y^{n}}{q+y^{n}}+\epsilon Q_{p}\left(T_{o n}, \Delta t\right) . Q_{p}\left(T_{o n}, \Delta t\right)$ is the square pulse function of unit height starting at $t=T_{\text {on }}$ and of duration $\Delta t$, while $\epsilon$ is the perturbation amplitude. When the amplitude and duration of the perturbation are below the corresponding thresholds the population of the semidifferentiated cells increases while the perturbation is on, then it returns back to its stationary value after some variable time interval. If the $\epsilon$ (or $\Delta t$ ) value is greater than the threshold then unlimited growth occurs (middle panel of Fig. 4).

Let us now consider the case in which the semidifferentiated cell population is subject to random fluctuations described by $\sigma \xi(t)$. We are interested in investigating the effects of these fluctuations on the time evolution of the cell populations. A typical trajectory describing the time evolution of the population size of the semidifferentiated cells is shown in the bottom panel of Fig. 4. Initially the population size fluctuates around the value of $y_{e q}$ corresponding to the stationary state $S_{1}$, then the trajectory enters the basin of attraction of the unstable state $S_{2}$ and unlimited growth occurs. To characterize the behavior of the dynamical system the probability $P\left(S_{1} \rightarrow S_{2}\right)$ of entering the basin of attraction of the unstable state was estimated numerically for several parameter values. The probability was evaluated as $N_{u} / N_{\text {Trials }}$, where $N_{u}$ is the total number of times that the attraction basin of $S_{2}$ is crossed by starting from $S_{1} ; N_{\text {Trials }}$ is the total number of trajectories starting from $S_{1}$. Several numerical experiments were carried out with different values of $N_{\text {Trials }}$ and they showed that the choice adopted here $\left(N_{\text {Trials }}=5000\right)$ is an acceptable compromise between statistical precision and computational cost. The left panel of Fig. 5 shows the values of $P\left(S_{1} \rightarrow S_{2}\right)$ plotted against the noise amplitude for different parameter values. The probability of a transition to $S_{2}$ increases in a sigmoidal fashion as the noise
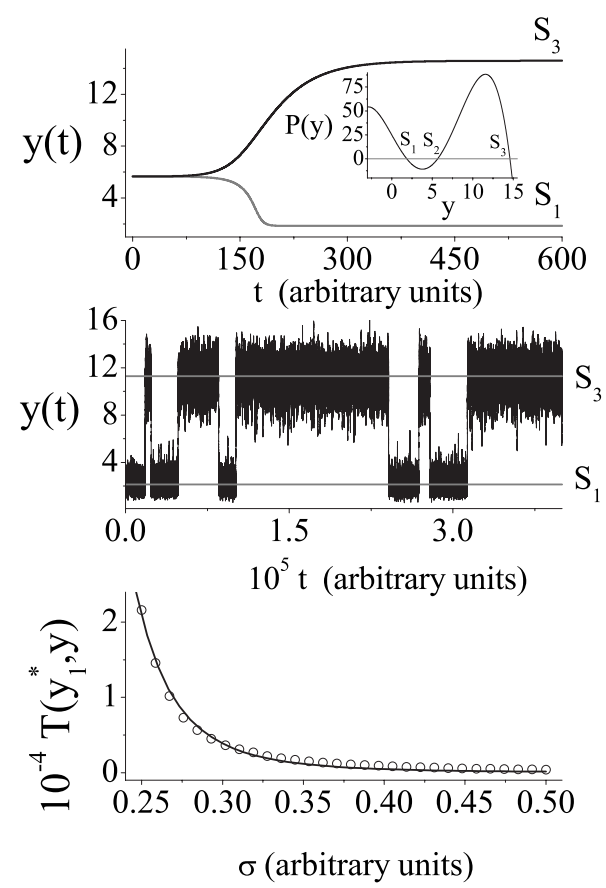

FIG. 6. Top panel: time evolution of the population of semidifferentiated cells starting with slightly different initial conditions from the unstable state $S_{2}$ in absence of noise. In the inset is reported the plot of $P(y)$ corresponding to the following parameter values: $\alpha=0.5, \alpha_{2}=0.1, k=0.1, \beta=-0.3, p=0.27, q=50, n=3$. Middle panel: a typical time evolution of the population of semidifferentiated cells in the presence of noise of amplitude $\sigma=0.25$. Bottom panel: mean switching time from $S_{1}$ to $S_{3}$ estimated by solving numerically Eqs. (3a)-(3c) (open circles) against the noise amplitude; the values of the mean switching time computed analytically are represented by the black line. For all panels $p+\beta>0$.

amplitude increases. The decrease in the $p$ value $(0<\beta+p)$ requires higher noise amplitudes for unlimited growth (thin black line). Keeping $p$ fixed (0.35), the decrease in the selfrenewal rate of the stem cell population strongly impacts the values of the transition probability $P\left(S_{1} \rightarrow S_{2}\right)$ (gray line). Taken together these results indicate that when there is a perturbation of the process driving cellular proliferation the presence of noise can determine a transition toward unlimited tissue growth. The left panel of Fig. 5 shows the mean exit time $\left\langle T\left(S_{1}\right)\right\rangle$ plotted against the initial value of the population size of the semidifferentiated cells for different noise amplitudes. The value of the last quantity was estimated numerically as $\left\langle T\left(S_{1}\right)\right\rangle=\frac{1}{N_{u}} \sum_{j=1}^{N_{u}} T_{j}$, where $T_{j}$ is the time required to move from $S_{1}$ to $S_{2}$. The results show, as expected, that there is a decrease in the mean exit time for increasing values of the initial population size. Moreover the increase in the noise amplitude has a strong influence on the mean exit time values: the higher the $\sigma$, the shorter is the corresponding $\left\langle T\left(S_{1}\right)\right\rangle$. Let us now consider the case in which $(\beta+p)<0$ and $n$ takes odd values. The adopted parameter values are $\alpha=0.5, \alpha_{2}=0.1, k=0.1, \beta=-0.3, p=0.27, q=50, n=3$ and the corresponding roots of the polynomial $P(y)$ are $y_{1}^{*}$ $\cong 1.86, y_{2}^{*} \cong 5.66, y_{3}^{*} \cong 14.6$. The plot of the corresponding polynomial is shown in the inset in the top panel of Fig. 6. This choice of parameters corresponds to the situation de- 
scribed in Fig. 2(d), where there are three stationary states $S_{1}, S_{2}, S_{3}$ of Eqs. (3a)-(3c): $S_{1}, S_{3}$ are stable, while $S_{2}$ is unstable. The top panel of Fig. 6 shows that, in absence of noise $(\sigma=0)$, starting with initial conditions close to $S_{2}$ the time evolution of the population of semidifferentiated cells can reach $S_{1}$ or $S_{3}$. In this case therefore there is bistability: i.e., the dynamical system evolves toward one of the two stable stationary states depending on the initial conditions. The middle panel of Fig. 6 shows a typical trajectory occurring when $\sigma \neq 0$ and shows that the presence of noise determines a switching from the stable state $S_{1}$ to $S_{3}$ and vice versa. To determine the mean switching time between these states and how it depends on the noise intensity we consider here the case in which at $t=0$ the state of the dynamical system is $S_{1}$. We use numerical simulations to estimate how the mean switching time $T_{s}\left(S_{1} \rightarrow S_{3}\right)$ depends on the noise amplitude. For a fixed value of $\sigma$ the system of differential equations was integrated numerically up to $T_{\text {Int }}=40 \times 10^{6}$. Then from this trajectory the residence time intervals $\Delta t_{S_{1}}(j)$ $(j=1,2, \ldots, M)$ in the state $S_{1}$ were computed and from them the mean switching time from $S_{1}$ to $S_{3}$ was estimated as $T_{s}\left(S_{1} \rightarrow S_{3}\right)=\frac{1}{M} \sum_{j} \Delta t_{S_{1}}(j)$. The results are shown in the bottom panel of Fig. 6 (open circles). The increase in the noise amplitude determines a very fast shortening of the mean switching time. To characterize the switching phenomenon occurring in this dynamical regime a theoretical analysis was carried out. First of all let us assume that the population of stem cells is at equilibrium: i.e., $x(t)=x_{e q}=\alpha / k$. This assumption makes sense because all simulations were performed by starting from the stationary state $S_{1}$. However no significant changes occur if $x(t) \neq x_{e q}=\alpha / k$ : it is just sufficient that the sampling of the numerical trajectories occurs after $t \gg \alpha^{-1}$. Under this condition the time evolution of the population of the semidifferentiated cells is described by the following equation: $\dot{y}=-\frac{d U(y)}{d y}+\sigma \xi(t)$, with $U(y)=-\int[\beta y+R+F(y) y] d y$. From the theory of stochastic differential equations it follows that the mean exit time from the interval $\left[y_{1}^{*}, y\right]$ when all trajectories start from $y_{1}^{*}$ is given by $T\left(y_{1}^{*}, y\right)$ $=\frac{1}{\sigma^{2}} \int_{y_{1}^{*}}^{y} d y^{\prime} e^{U\left(y^{\prime}\right) / \sigma^{2}} \int_{-\infty}^{y^{\prime}} d z e^{-U(z) / \sigma^{2}}[63]$. The values of $T\left(y_{1}^{*}, y\right)$ were computed numerically for the case $n=3$ and the corresponding results are shown in the bottom panel of Fig. 6 (black line). The comparison of the theoretical results $\left[T\left(y_{1}^{*}, y\right)\right.$ values $]$ with those obtained in the numerical experiments show good agreement.

Summarizing, the above results indicate that the alteration of the proliferative activity of the semidifferentiated cells [described by $\left.p y^{n+1} /\left(q+y^{n}\right)\right]$ can promote bistable behavior in the cellular population. Moreover the presence of noise of suitable amplitude determines a switching between these stable states. Other numerical simulations were carried out by using different parameter values. The corresponding results are qualitatively similar to those already discussed (results not shown).

\section{CONCLUSION}

In this paper a continuous nonlinear model describing the cell populations in the colonic crypts was studied analyti- cally and numerically. Three cell types were considered: the stem cells, the semidifferentiated cells, and the fully differentiated cells. In the first part of the paper we studied how an alteration of the renewal rate of the semidifferentiated cells affects the growth properties of these cell populations. To this aim it was assumed that the rate of the self-renewal of the semidifferentiated cells was $\beta_{3}(y)=\beta_{3}+\frac{p y^{n}}{q+y^{n}}\left(\beta=\beta_{3}-\beta_{2}\right.$ $-\beta_{1}$ ). It was found that unlimited growth occurs if $-p<\beta$ $<0$. Moreover when $\beta<-p$ two possible behaviors can occur: a single stable stationary state or bistability. The above investigations were extended by studying the more general case $\beta_{3}(y)=\beta_{3}+F(y) \quad$ with $F(0)=0, \quad F^{\prime}(y)>0$, and $\lim _{y \rightarrow+\infty} F(y)=L<+\infty$. It was shown that when $L<-\beta$ there exists at least one stationary state of the dynamical system. Moreover it was shown that when there exists only one stationary state then it is globally stable. It was proved that the above results also hold when the function $F(y)$ satisfies weaker constraints. The last part of the paper was devoted to the discussion of the results obtained with numerical simulations. In particular it was shown that the presence of the noise can lead to unlimited growth of the population of the semidifferentiated cells. Moreover bistability phenomena were found and characterized quantitatively.

\section{ACKNOWLEDGMENTS}

A.D.G. wishes to acknowledge the Centre for Mathematical Biology of the Mathematical Institute of the University of Oxford for its kind hospitality and the Italian National Research Council (CNR) for financial support. M.D.J. was supported by a DTA Graduate Studentship (Award No. EP/ P500397/1) from the EPSRC and by NIH (NCI) Virtual Tumor Grant No. U56 CA 113004-02. P.K.M. was partially supported by a Royal Society-Wolfson Research Merit Award and NIH (NCI) Virtual Tumor Grant No. U56 CA 113004-02.

\section{APPENDIX A: PROOF OF PROPOSITION II}

The function $\left(\frac{R}{y}+\beta\right)$ crosses the $y$ axis at $y_{1}=-R / \beta$. Moreover, let $y_{2}=-R /(\beta+L)>0$ be the solution of the equation $-\left(\frac{R}{y}+\beta\right)=L$. Let us define $G(y)=F(y)+\left(\frac{R}{y}+\beta\right)$ : this is differentiable and continuous in the interval $] 0,+\infty[$. Let us now prove that $G(y)>0$ for $y \in] 0, y_{1}\left[=I_{1}\right.$. From the inequalities $F(y)>0$ and $(R / y+\beta)>0$ for $y \in I_{1}$ it follows that $G(y)>0$. Let us now prove that $G(y)<0$ for $y \in] y_{2},+\infty[$ $=I_{2}$. The inequality $L<-\beta$ implies that $(R / y+\beta)<-L$ for $y>y_{2}$. Then, from $0<F(y)<L$ for $y \in I_{2}$ it follows that $G(y)<0$ in $I_{2}$. Now, since $G(y)>0$ in $I_{1}$ and $G(y)<0$ in $I_{2}$, the Bolzano theorem implies that the function $G(y)$ possesses at least a root $y^{*}$ in the interval $] y_{1}, y_{2}[$.

\section{APPENDIX B: PROOF OF PROPOSITION III}

Proof. The eigenvalues of the Jacobian matrix evaluated at $S_{0}=\left(\frac{\alpha}{k}, y^{*}, \frac{\beta_{2} y^{*}}{\gamma}\right)$ are $\lambda_{1}=-\alpha<0, \lambda_{2}=\beta+\frac{d}{d y}[y F(y)]_{y=y^{*}}, \lambda_{3}$ $=-\gamma<0$. To show that $S_{0}$ is a linearly stable stationary state it is sufficient to show that $\lambda_{2}<0$ : i.e., $\lambda_{2}=\beta+F\left(y^{*}\right)$ $+y^{*}\left[\frac{d F(y)}{d y}\right]_{y=y^{*}}=-R / y^{*}+y^{*}\left[\frac{d F(y)}{d y}\right]_{y=y^{*}}<0$. Since $y^{*}>0$ it fol- 
lows that the previous inequality is equivalent to $\left[\frac{d F(y)}{d y}\right]_{y=y^{*}}$ $<R / y^{* 2}$. Let $g(y)=-(R / y+\beta)$ and $y_{1}=-R / \beta$, then $g\left(y_{1}\right)=0$ and $F\left(y_{1}\right)>g\left(y_{1}\right)$. Moreover from proposition II it follows that $y^{*}>y_{1}$. Thus, $F(y)>g(y)$ in the interval $\left[y_{1}, y^{*}[\right.$ because $y^{*}$ is the only root of the equation $F(y)-g(y)=0$. Now let $0<h \ll 1$, then $F\left(y^{*}-h\right)=F\left(y^{*}\right)-h\left[\frac{d F(y)}{d y}\right]_{y=y^{*}}+o(h)$ and $g\left(y^{*}\right.$ $-h)=g\left(y^{*}\right)-h R / y^{* 2}+\bar{o}(h)$. Now since $F(y)>g(y)$ in $\left[y_{1}, y^{*}[\right.$ it follows that $-h\left[\frac{d F(y)}{d y}\right]_{y=y^{*}}+o(h)>-h R / y^{* 2}+\bar{o}(h)$. Dividing both sides by $h$, and taking the limit for $h \rightarrow 0$, it follows that $\left[\frac{d F(y)}{d y}\right]_{y=y^{*}}<-R / y^{* 2}$. Let us now show that the previous inequality also holds when a right neighbor of $y^{*}$ is considered. Let $\left.I_{1}=\right] y^{*}, y^{*}+\epsilon$ [ with $0<\epsilon \ll 1$ and $y^{*}+\epsilon<y_{2}=-R /(\beta+L)$. From proposition II it follows that $F(y)<g(y)$ for $y$ $\in] y_{2}, \infty\left[\right.$. If $F(y)>g(y)$ in $I_{1}$ then in the interval $] y^{*}+\epsilon, y_{2}[$ at least one other root can be found. But this contradicts the hypothesis of the proposition and therefore $F(y)<g(y)$ in $I_{1}$. Now let $0<h<\epsilon \ll 1$; then, since $F(y)<g(y)$ in $I_{1}$, it follows that the expansion of $F\left(y^{*}+h\right)<g\left(y^{*}+h\right)$ leads to $\left[\frac{d F(y)}{d y}\right]_{y=y^{*}}<-R / y^{* 2}$, and this implies $\lambda_{2}<0$. As $S_{0}$ $=\left(x_{e q}, y_{e q}, z_{e q}\right)=\left(\frac{\alpha}{k}, y^{*}, \frac{\beta_{2} y^{*}}{\gamma}\right)$ is a hyperbolic fixed point it follows, from the Hartman-Grobman theorem, that the linear asymptotic stability implies nonlinear asymptotic stability.

\section{APPENDIX C: PROOF OF PROPOSITION IV}

Unlimited growth implies that $y(t)$ is unbounded: i.e., $d y(t) / d t>0$ in a positive neighborhood of $y_{N}^{*}$ and then $y(t)$ grows indefinitely. This implies that the stationary state corresponding to $y_{N}^{*}$ is unstable.

\section{APPENDIX D: PROOF OF PROPOSITION V}

Proof. From $\beta+L<0$ it follows that $\lim _{y \rightarrow+\infty} \frac{d y}{d t}=-\infty$. Then there exists $y^{*} \in \mathfrak{R}^{+}$such that $\frac{d y}{d t}<0$ for $y>y^{*}$. To show that the solutions of Eqs. 16 and 17 are bounded it is sufficient to prove that for any initial condition $[y(0), z(0)]$ $=\left(y_{I}, z_{I}\right)$, with $y_{I}>y^{*} z_{I}>0$ the corresponding solutions cannot grow indefinitely. As $y_{I}>y^{*}$ it follows that $y(t) \leq y_{I}$ for $t \geq 0$. Equation (9c) implies that $z(t)=e^{-\gamma t}\left[z_{I}\right.$ $\left.+\int_{0}^{t} \beta_{2} y\left(t^{\prime}\right) e^{\gamma t^{\prime}} d t^{\prime}\right]$. As $0 \leq y(t) \leq y_{I}$ it follows that $0 \leq z(t)$ $\leq e^{-\gamma t}\left[z_{I}+\beta_{2} y_{I} \int_{0}^{t} e^{\gamma t^{\prime}} d t^{\prime}\right]=e^{-\gamma t}\left[z_{I}-1 / \gamma\right]+\left(\beta_{2} y_{I}\right) / \gamma=r(t)$. As $r(t)$ is a bounded function it follows that $z(t)$ will also be a bounded function.
[1] B. Boman and E. Huang, J. Clin. Oncol. 26, 2828 (2008).

[2] S. Majumder, Stem Cells and Cancer (Springer-Verlag, New York, 2009).

[3] P. Kaur and C. Potten, Cell Prolif 19, 601 (1986).

[4] J. Heath, Cell Biol. Int. 20, 139 (1996).

[5] L. van der Flier and H. Clevers, Annu. Rev. Physiol. 71, 241 (2009).

[6] I. Tomlinson and W. Bodmer, Proc. Natl. Acad. Sci. U.S.A. 92, 11130 (1995).

[7] B. Boman, J. Fields, O. Bonham-Carter, and O. Runquist, Cancer Res. 61, 8408 (2001).

[8] K. Hardy and J. Stark, Apoptosis 7, 373 (2002).

[9] B. Deasy, R. Jankowski, T. Payne, B. Cao, J. Goff, J. Greenberger, and J. Huard, Stem Cells 21, 536 (2003).

[10] F. Michor, Y. Iwasa, H. Rajagopalan, C. Lengauer, and M. Nowak, Cell Cycle 3, 356 (2004).

[11] N. Komarova and L. Wang, Cell Cycle 3, 1558 (2004).

[12] R. Ganguly and I. Puri, Cell Prolif 39, 3 (2006).

[13] I. van Leeuwen, H. Byrne, O. Jensen, and J. King, Cell Prolif 39, 157 (2006).

[14] D. Wodarz, J. Theor. Biol. 245, 449 (2007).

[15] I. van Leeuwen, H. Byrne, O. Jensen, and J. King, J. Theor. Biol. 247, 77 (2007).

[16] F. Michor, J. Clin. Oncol. 26, 2854 (2008).

[17] M. Johnston, C. Edwards, W. Bodmer, P. K. Maini, and S. Chapman, Proc. Natl. Acad. Sci. U.S.A. 104, 4008 (2007).

[18] A. d'Onofrio and I. P. M. Tomlinson, J. Theor. Biol. 244, 367 (2007).

[19] W.-C. Lo, C.-S. Chou, K. K. Gokoffski, F. Y.-M. Wan, A. D. Lander, A. L. Calof, and Q. Nie, Math. Biosci. Eng. 6, 59 (2008).

[20] N. Komarova, Semin Cancer Biol. 15, 494 (2005).

[21] P. Nowell, Semin Cancer Biol. 12, 261 (2002).
[22] N. Bellomo, N. Li, and P. K. Maini, Math. Models Meth. Appl. Sci. 18, 593 (2008).

[23] P. Nowell, Science 194, 23 (1976).

[24] J. Murray, Mathematical Biology (Springer-Verlag, New York, 1989).

[25] F. Sproull and C. David, J. Cell. Sci. 38, 155 (1979).

[26] V. Zhdanov, Chem. Phys. Lett. 437, 253 (2007).

[27] H. Kilian, D. Bartkowiak, D. Kaufmann, and R. Kemkemer, Cell Biochem. Biophys. 51, 51 (2008).

[28] L. Mancuso et al., Cell Prolif 42, 602 (2009).

[29] A. d'Onofrio, Physica D 208, 220 (2005).

[30] B. Q. Ai, X. J. Wang, G. T. Liu, and L. G. Liu, Phys. Rev. E 67, 022903 (2003).

[31] A. Laird, Br. J. Cancer 19, 278 (1965).

[32] P. Lala and H. Patt, Proc. Natl. Acad. Sci. U.S.A. 56, 1735 (1966).

[33] L. Norton, Cancer Res. 48, 7067 (1988).

[34] J. Spratt, D. von Fournier, J. Spratt, and E. Weber, Cancer 71, 2013 (1993).

[35] C. Potten, C. Booth, and D. Hargreaves, Cell Prolif 36, 115 (2003).

[36] S. Leedham, M. Brittan, S. McDonald, and N. Wright, J. Cell. Mol. Med. 9, 11 (2005).

[37] M. Mackey, Cell Prolif 34, 71 (2001).

[38] M. Johnston, C. Edwards, W. Bodmer, P. K. Maini, and S. Chapman, Cell Cycle 6, 2106 (2007).

[39] J. Guckenheimer and P. Holmes, Nonlinear Oscillations, Dynamical System, and Bifurcations of Vector Fields (SpringerVerlag, New York, 1983).

[40] T. Enver, M. Pera, C. Peterson, and P. Andrews, Cell Stem Cell 4, 387 (2009).

[41] J. Hasty, J. Pradines, M. Dolnik, and J. Collins, Proc. Natl. Acad. Sci. U.S.A. 97, 2075 (2000). 
[42] K. Lai, M. Robertson, and D. Schaffer, Biophys. J. 86, 2748 (2004).

[43] S. Huang, G. Eichler, Y. Bar-Yam, and D. E. Ingber, Phys. Rev. Lett. 94, 128701 (2005).

[44] H. Chang, P. Oh, D. Ingber, and S. Huang, BMC Cell Biol. 7, 11 (2006).

[45] J. Gurdon and D. Melton, Science 322, 1811 (2008).

[46] V. Zhdanov, Biosystems 95, 75 (2009).

[47] L. Wang, B. Walker, S. Iannaccone, D. Bhatt, P. Kennedy, and W. Tse, Proc. Natl. Acad. Sci. U.S.A. 106, 6638 (2009).

[48] H. Chang, M. Hemberg, M. Barahona, D. Ingber, and S. Huang, Nature (London) 453, 544 (2008).

[49] C. David and H. MacWilliams, Proc. Natl. Acad. Sci. U.S.A. 75, 886 (1978).

[50] A. Chatterjee, Y. Kaznessis, and W. Hu, Curr. Opin. Biotechnol. 19, 475 (2008).

[51] R. Guantes and J. Poyatos, PLOS Comput. Biol. 4, e1000235 (2008).

[52] J. Ferrell, Curr. Opin. Cell Biol. 14, 140 (2002).

[53] W. Xiong and J. Ferrell, Nature (London) 426, 460 (2003).
[54] G. Yao, T. Lee, S. Mori, J. Nevins, and L. You, Nat. Cell Biol. 10, 476 (2008).

[55] Y. Chai, F. Chu, R. Kolter, and R. Losick, Mol. Microbiol. 67, 254 (2008).

[56] A. Gordon, J. Halliday, M. Blankschien, P. Burns, F. Yatagai, and C. Herman, PLoS Biol. 7, e1000044 (2009).

[57] C. Rao, D. Wolf, and A. Arkin, Nature (London) 420, 231 (2002).

[58] W. Blake, M. Kaern, C. Cantor, and J. Collins, Nature (London) 422, 633 (2003).

[59] M. Hoffmann, H. Chang, S. Huang, D. Ingber, M. Loeffler, and J. Galle, PLoS ONE 3, e2922 (2008).

[60] T. Matsui, S. Tsuda, K. Yao, A. Iwashita, T. Sakurai, and T. Yao, Dis. Colon Rectum 43, S18 (2000).

[61] M. Al-Hajj and M. Clarke, Oncogene 23, 7274 (2004).

[62] M. Alison, G. Murphy, and S. Leedham, Cell Tissue Res. 331, 109 (2008).

[63] C. Gardiner, Stochastic Methods (Springer-Verlag, New York, 2009). 